This paper has been published in Annali di Matematica Pura ed Applicata. Series IV, 189(4):567-570 (2010).

Copyright 2010 by Springer-Verlag.

The final publication is available at www.springerlink.com.

http://link. springer.com/article/10.1007\%2Fs10231-009-0124-7

http://dx.doi.org/10.1007/s10231-009-0124-7 


\title{
On a graph related to permutability in finite groups
}

\author{
A. Ballester-Bolinches* John Cossey ${ }^{\dagger} \quad$ R. Esteban-Romero ${ }^{\ddagger}$
}

\begin{abstract}
For a finite group $G$ we define the graph $\Gamma(G)$ to be the graph whose vertices are the conjugacy classes of cyclic subgroups of $G$ and two conjugacy classes $\mathcal{A}, \mathcal{B}$ are joined by an edge if for some $A \in \mathcal{A}$, $B \in \mathcal{B} A$ and $B$ permute. We characterise those groups $G$ for which $\Gamma(G)$ is complete.

Mathematics Subject Classification (2000): 5C25, 20D15, 20D20,

Keywords: Finite groups, graphs, soluble groups, permutability
\end{abstract}

\section{Introduction}

There are many ways in which a graph has been associated with a finite group. Herzog, Longobardi and Maj [8] have defined a graph whose vertices are the conjugacy classes of a group, with two vertices joined by and edge if an element of one vertex commutes with some element of the other vertex. This is a generalisation of the commuting graph of a group, which has the elements of a group as vertices, joined by an edge if they commute (see [9]). In this paper we will consider a generalisation of the graph of Herzog, Longobardi and Maj. For a finite group $G$ we define the graph $\Gamma(G)$ to be the graph whose vertices are the conjugacy classes of cyclic subgroups of $G$ and two conjugacy classes $\mathcal{A}, \mathcal{B}$ are joined by an edge if for some $A \in \mathcal{A}$, $B \in \mathcal{B} A$ and $B$ permute. We characterise those groups $G$ for which $\Gamma(G)$ is complete.

\footnotetext{
*Departament d'Àlgebra, Universitat de València; Dr. Moliner, 50; 46100, Burjassot, València, Spain; email: Adolfo.Ballester@uv.es

${ }^{\dagger}$ Mathematics Department, Mathematical Sciences Institute, Australian National University; Canberra, ACT 0200, Australia; email: John.Cossey@anu.edu.au

${ }^{\ddagger}$ Institut Universitari de Matemàtica Pura i Aplicada, Universitat Politècnica de València; Camí de Vera, s/n; 46022 València, Spain, email: resteban@mat.upv.es
} 
Recall that a subgroup $H$ of a group $G$ is said to be permutable in $G$ if $H L$ is a subgroup of $G$ for every subgroup $L$ of $G$. Permutability, like normality, is not a transitive relation in general. We say that a group $G$ is a $P T$-group if the permutability is transitive in $G$, that is, if $H$ is permutable in $K$ and $K$ is permutable in $G$, then $H$ is permutable in $G$. According to a classical result of Ore [10] permutable subgroups of finite groups are subnormal. Hence a finite group is a PT-group if and only if every subnormal subgroup is permutable.

We prove:

Theorem 1. A finite group $G$ is a soluble PT-group if and only if the graph $\Gamma(G)$ is complete.

\section{Proof of Theorem 1}

Suppose that $G$ is a soluble PT-group. By a result of Zacher [12] $G=A H$ where $A$ is an abelian normal subgroup of $G, H$ is a nilpotent modular subgroup of $G, A$ and $H$ have coprime orders and every subgroup of $A$ is normal in $G$. If $X$ and $Y$ are two cyclic subgroups of $G$, we can write $X=X_{0} X_{1}$ and $Y=Y_{0} Y_{1}$, where $X_{0}$ and $Y_{0}$ are subgroups of $A$ and $X_{1}$ and $Y_{1}$ have orders dividing $|H|$. Since $H$ is a Hall subgroup of $G$ we can, by replacing $X$ and $Y$ by conjugates if necessary, assume that $X_{1}$ and $Y_{1}$ are subgroups of $H$. Since every subgroup of $A$ is normal in $G$ we have $Y_{0} X_{1}=X_{1} Y_{0}$ and since $H$ is modular we have $X_{1} Y_{1}$ is a subgroup of $G$. It now follows that $X_{0} X_{1} Y_{0} Y_{1}=Y_{0} Y_{1} X_{0} X_{1}$ is a subgroup, that is, $X$ and $Y$ permute.

In the other direction, we argue by induction on the order of $G$. We begin by showing that $G$ is a soluble PT-group if $G$ has at least two minimal normal subgroups. If $M$ and $N$ are two minimal normal subgroups of $G$, then $G / M$ and $G / M$ clearly satisfy the hypothesis of the theorem. Hence $G / M$ and $G / N$ are soluble PT-groups. It follows that $N M / M$ is a minimal normal subgroup of a soluble PT-group, and so is cyclic of prime order because every soluble PT-group is supersoluble. Similarly $M N / N$ is cyclic of prime order and hence $M$ and $N$ have prime orders, $p$ and $q$ say (and $G$ is soluble). Then, for any prime $r \neq p$, all $r$-chief factors of $G / M$ are $G$-isomorphic. Further, by Zacher's Theorem [12], Sylow $r$-subgroups of $G / M$ are abelian if $r$-chief factors are noncentral and modular if all $r$-chief factors are central. If $p \neq q$ by considering $G / N$ we have all $p$-chief factors $G$-isomorphic and Sylow $p$-subgroups abelian if $p$-chief factors are noncentral and modular if $p$-chief factors are central. In this case $G$ is a PT-group by [3, Corollary 3] and [4, Theorem 2] (note that $G$ is supersoluble). 
Thus we suppose that all minimal normal subgroups have the same prime order $p$. If $M$ and $N$ are minimal normal subgroups and $p$ divides $|G / M N|$, then both $M$ and $N$ are $G$-isomorphic to a (fixed) $p$-chief factor of $G / M N$ and so are $G$-isomorphic. Thus all $p$-chief factors are $G$-isomorphic. Therefore $G$ is supersoluble and all chief factors of the same order are $G$-isomorphic. By [3, Corollary 3] $G$ is a group in which every subnormal subgroup permutes with all Sylow subgroups ( $G$ is a PST-group). If the $p$-chief factors are central, then $G$ is a $p$-group with all proper quotients modular and so is itself modular, since by Theorem of Longobardi [7] such a group must have a unique minimal normal subgroup. Applying a result of Agrawal [2], $G$ has an abelian Sylow $p$-subgroup and it then follows that $G$ is a PT-group by [4, Theorem 2]. Assume now that $M N$ is a Sylow $p$-subgroup of $G$. Let $M=\langle m\rangle, N=\langle n\rangle$. By hypothesis, given a $p^{\prime}$-element $y \in G,\langle m n\rangle$ permutes with a conjugate $\left\langle y^{g}\right\rangle$ of $\langle y\rangle$. Hence $\langle m n\rangle\left\langle y^{g}\right\rangle \cap M N=\langle m n\rangle$ is normalised by $y^{g}$. Call $m^{g}=m^{a_{1}}, n^{g}=m^{a_{2}}$ and $m^{y}=m^{b_{1}}, n^{y}=n^{b_{2}}$ and $(m n)^{g^{-1} y g}=(m n)^{c}$. Hence $(m n)^{g^{-1} y g}=m^{b_{1}} n^{b_{2}}=(m n)^{c}$, which implies that $b_{1} \equiv b_{2} \equiv c(\bmod p)$. Consequently $M$ and $N$ are $G$-isomorphic. Since $G$ has all Sylow subgroups modular, it follows that $G$ is a PT-group by [3, Corollary 3] and [4, Theorem 2].

We now suppose that $G$ has a unique minimal normal subgroup $N$. If $N$ is not soluble, then $N=S_{1} \times \cdots \times S_{r}$, where the $S_{i}$ are isomorphic (nonabelian) simple groups. Let $p$ and $q$ be different primes dividing the order of $S_{1}$ and let $x_{1}$ and $y_{1}$ be elements of $S_{1}$ of orders $p$ and $q$, respectively. For $2 \leq i \leq r$, let $x_{i}, y_{i}$ be the images of $x_{1}, y_{1}$ under the isomorphism between $S_{1}$ and $S_{i}$. Then $\left\langle x_{1} \cdots x_{r}\right\rangle$ permutes with a conjugate $\left\langle\left(y_{1} \cdots y_{r}\right)^{g}\right\rangle$ of $\left\langle y_{1} \cdots y_{r}\right\rangle$. The projection of $\left\langle x_{1} \cdots x_{r}\right\rangle\left\langle\left(y_{1} \cdots y_{r}\right)^{g}\right\rangle$ onto $S_{1}$ is then a subgroup of $S_{1}$ of order $p q$ and so $S_{1}$ has subgroups of order $p q$ for every pair of primes dividing its order. A result of Abe and Iiyori [1] shows that this is impossible. Consequently $N$ is a $p$-group for some prime $p$.

If $N$ is not contained in the Frattini subgroup of $G$, then $G$ is a primitive soluble group and $G=N M$, where $M$ is a maximal subgroup of $G, N \cap M=1$ and $N$ is self-centralising. Since $M$ is isomorphic to the soluble PT-group $G / N, M$ is the product of its nilpotent residual $F=M^{\mathfrak{N}}$, which is an abelian normal Hall subgroup of odd order, and a complement $C$ which acts on $F$ as power automorphisms ([12]). Let $Q$ be a cyclic normal subgroup of $M$. Suppose that $Q P \neq P Q$ for some cyclic subgroup $P$ of $N$. We have $Q^{g} P=P Q^{g}$ for some $g \in G$ and we can assume that $g \in M$. Since $Q$ is normal in $M$ we have $Q^{g}=Q$, giving a contradiction. Thus $P$ is normalised by $Q$ since $P=N \cap P Q$. It now follows that every element of $F$ acts as a power automorphism on $N$ and hence $F$ acts as a power automorphism group on $N$. Since power automorphisms are central in the power automorphism 
group of $N$ ([5, Theorem 2.2.1]), $F$ is central in $M$ and so $F=1$. Thus $M$ is a nilpotent modular group. In particular $M$ is a $p^{\prime}$-group. Let $Q$ be a non-abelian Sylow $q$-subgroup of $M$. By Iwasawa's Theorem ([11, Theorem 2.4.14] $Q$ has an abelian normal subgroup $Q_{0}$ with cyclic supplement $S$ with $S$ acting as a power automorphism group on $Q_{0}$ or $Q$ is Hamiltonian. In both cases every cyclic subgroup of $Q_{0}$ is normal in $Q$ and hence in $M$. Let $U$ be a cyclic subgroup of $N$ and let $R$ cyclic subgroup of $Q_{0}$. By hypothesis, there exits an element $a \in M$ such that $R U^{a}$ is subgroup. Since $R^{a}=R$, we have that $R U$ is also a subgroup and $U$ is normalised by $R$. Further, there exists an element $m \in M$ such that $S$ permutes with $U^{m}$ and so $S$ and $R$ normalise $U^{m}$. This implies that $Q$ normalises $U$ and $Q$ acts as power automorphisms on $N$. It now follows that $M$ acts as power automorphisms on $N$ and so $N$ is a cyclic group of order $p$ and $M$ is cyclic of order dividing $p-1$ and $G$ is clearly a PT-group.

Now suppose that $N$ is contained in the Frattini subgroup of $G$. If $G$ is nilpotent, then $G$ is a $p$-group since it has a unique minimal normal subgroup and $G / N$ is an modular group. Assume that $G$ is not modular. Let $M(p)$ denote the nonabelian group of order $p^{3}$ and exponent $p$ for $p$ odd and the dihedral group of order 8 for $p=2$. By the Theorem of Longobardi [7] either $G$ is the central product of a subgroup $P$ isomorphic to $M(p)$ and another subgroup or $G$ is isomorphic to

$$
G_{0}=\left\langle a, b, w: a^{p^{n}}=w^{p}=1, a^{b}=a^{1+p^{s}}, b^{p^{j}}=a^{p^{n-s}}, a^{w}=a^{1+p^{n-1}}, b^{w}=b\right\rangle,
$$

where $0<s<n, s \geq 2$ if $p=2$ and $j \geq n-s$. In the first case it is clear that if $P$ is generated by $a$ and $b$ of order $p$ no conjugate of $a$ will commute with $b$ and hence will not permute with $b$. Now consider $G_{0}$ and let $C=\left\langle a^{\alpha} b^{\beta}\right\rangle$ be a cyclic subgroup of $H=\langle a, b\rangle$. Then $C$ permutes with $\left\langle w^{g}\right\rangle$ for some $g \in G_{0}$ and so, being of index $p$ in $C\left\langle w^{g}\right\rangle$, is normalised by $w^{g}$. Since $[w, g] \in\left\langle a^{p^{n-1}}\right\rangle, C$ is also normalised by $w$. Thus $w$ acts as a power automorphism on $H$. If $p$ is odd, $H$ is regular ([6, III, Satz 11.4]) and so it acts as a universal power automorphism on $H$ by [5, Theorem 5.3.1], a contradiction. Hence we suppose $p=2$. Since $w$ centralises $b$ and $b$ has order at least $4, w$ acts as universal power automorphism on $H$ by a theorem of Napolitani [11, Theorem 2.3.24], again a contradiction. Thus $G$ cannot be nilpotent.

If $G$ is not nilpotent, then $E=G^{\mathfrak{N}} \neq 1$ and so $N \leq E$. Since $G / N$ is a PT-group, $G$ supersoluble, $E$ is nilpotent and so it is a $p$-group. Furthermore $E / N$ is abelian and complemented in $G / N$ by a $p^{\prime}$-subgroup $B / N$ say which acts on $E / N$ as power automorphisms. Then there exists a $p^{\prime}$-subgroup $D$ of $G$ complementing $E$ in $G$. If $C_{D}(E / N) \neq 1$ then $C_{D}(E / N)$ is a nontrivial 
normal subgroup of $G$, a contradiction. It follows that $D$ is cyclic of order dividing $p-1$. We have that $N \leq \mathrm{Z}(E)$. Consequently $\left[a^{p}, b\right]=[a, b]^{p}=1$ for every $a, b \in E$ by [6, III, Hilfssatz 1.3]. This implies that $E^{p} \leq \mathrm{Z}(E)$. Assume that $E^{p}$ is not trivial. Hence $E^{p}$ is cyclic because $E^{p}$ is an abelian normal subgroup of $G$. Let $E^{p}=\langle a\rangle$, where $a$ has order $p^{n}$. Suppose that $N$ is a proper subgroup of $E^{p}$. Given $x \in G$, we have that $a^{x}=a^{i}$ and so all chief factors of $G$ below $P^{p}$ are $G$-isomorphic. Appying again [3, Corollary 3] and [4, Theorem 2], $G$ is a PT-group. Assume now that $E^{p}=N$. Let $x$ be an element of $E$ not in $N$. If $x$ has order greater than $p$, then $N \leq\left\langle x^{p}\right\rangle \leq E^{p}$ and $\langle x\rangle / N$ is a normal subgroup of $G / N$. Since all chief factors of $G$ between $N$ and $E$ are $G$-isomorphic and the same happens with all chief factors of $G$ below $\langle x\rangle$, we conclude again that all chief factors of $G$ are $G$-isomorphic and $G$ is a PT-group. Thus all elements of $E$ are of order $p$. If $E$ is abelian then $E$ is cyclic and so $E=N \leq \Phi(G)$, a contradiction. Suppose that $E$ is non-abelian and $x, y \in E$ do not commute, so that $N=\langle[x, y]\rangle$. Since $\langle x\rangle$ permutes with $\left\langle y^{g}\right\rangle$ for some $g \in G$, we have that $\left\langle x, y^{g}\right\rangle$ is of order $p^{2}$ and hence abelian. Since $\langle y N\rangle$ is a normal subgroup of $G / N$, we have that $\left\langle y^{g}\right\rangle \leq\langle y, N\rangle$ and it follows that $y^{g}=y^{j} n$ for some $n \in N$ and some integer $j$ coprime with $p$. Then $\left[x, y^{g}\right]=\left[x, y^{j} n\right]=\left[x, y^{j}\right]=[x, y]^{j} \neq 1$, a contradiction. This completes the proof.

\section{Acknowledgements}

This paper has been suported by the research grant MTM2007-68010-C03-02 from MEC (Spain) and FEDER (European Union).

\section{References}

[1] S. Abe and N. Iiyori. A generalization of prime graphs of finite groups. Hokkaido Math. J., 29(2):391-407, 2000.

[2] R. K. Agrawal. Finite groups whose subnormal subgroups permute with all Sylow subgroups. Proc. Amer. Math. Soc., 47(1):77-83, 1975.

[3] M. J. Alejandre, A. Ballester-Bolinches, and M. C. Pedraza-Aguilera. Finite soluble groups with permutable subnormal subgroups. J. Algebra, 240(2):705-722, 2001.

[4] A. Ballester-Bolinches and R. Esteban-Romero. Sylow permutable subnormal subgroups of finite groups. J. Algebra, 251(2):727-738, 2002. 
[5] C. D. H. Cooper. Power automorphisms of a group. Math. Z., 107:335$356,1968$.

[6] B. Huppert. Endliche Gruppen I, volume 134 of Grund. Math. Wiss. Springer, Berlin, Heidelberg, New York, 1967.

[7] P. Longobardi. Gruppi finite a fattoriali modulari. Note Mat., II:73-100, 1982.

[8] P. Longobardi M. Herzog and M. Maj. On a commuting graph on conjugacy classes of groups. Commun. Algebra, 37(10):3369-3387, 2009.

[9] B. Neumann. A problem of Paul Erdôs on groups. J. Austral. Math. Soc. Ser. A, 21:467-472, 1976.

[10] O. Ore. Contributions to the theory of groups of finite order. Duke Math. J., 5:431-460, 1939.

[11] R. Schmidt. Subgroup lattices of groups, volume 14 of De Gruyter Expositions in Mathematics. Walter de Gruyter, Berlin, 1994.

[12] G. Zacher. I gruppi risolubli finiti in cui i sottogruppi di composizione coincidono con i sottogrupi quasi-normali. Atti Accad. Naz. Lincei Rend. cl. Sci. Fis. Mat. Natur. (8), 37:150-154, 1964. 